\title{
IMPROVING KNOWLEDGE OF TEXT NECK AND NECK PAIN USING INTERACTIVE SMARTPHONE APPLICATION FOR UNDERGRADUATE STUDENTS IN UNIVERSITI SAINS MALAYSIA
}

\author{
Jasni Dolah ${ }^{1}$ \\ Joey Loh Jo Yie ${ }^{2}$ \\ Lilian Lee Shiau Gee ${ }^{3}$ \\ ${ }^{1 \& 2}$ School of the Art, Universiti Sains Malaysia, Penang, Malaysia \\ ${ }^{3}$ Faculty of Social Sciences and Humanities, Universiti Malaysia Sabah, Sabah, Malaysia \\ jasnidolah@usm.my,joeymaster37@hotmail.com,lilian@ums.edu.my \\ Tarikh dihantar: 1 Julai 2020/Tarikh diterima: 13 Julai 2020
}

\begin{abstract}
Text neck syndrome has become more prevalent in modern life and can be avoided with proper neck warm-up exercise and frequent smartphone break-away. However, research has shown that due to a lack of education and awareness, most mobile users did not have the habit of break-away when using smartphones. Most solutions required the use of an external device attached to the body, resulting in significantly reduced ease of use and comfort. The objective of this study is to determine the level of knowledge and awareness of the text neck among undergraduate students of Universiti Sains Malaysia (USM) and to create an interactive mobile application to reduce the prevalence. The app is AR-based neck exercise gamification that alerts incorrect posture using a smartphone front camera and a built-in sensor. The proposed study will enable people who are suffering from, or at risk of, text neck to have more pleasant and engaging neck exercise experience with a better exercise impact, and may promote regular break-away from the wrong posture.
\end{abstract}

Keywords: Text neck, neck pain, interactive, mobile gamification.

\section{INTRODUCTION}

In modern life, Neck Pain (NP) and Text Neck (TN) or Turtle Neck Posture/Head Forward Posture (HFP) is becoming increasingly regular. Researchers found that TN has become a global epidemic affecting a sizeable smartphone-using population of almost all ages (Samani, Athavale, Shyam \& Sancheti, 2018; Vate-U-Lan, 2015) whereas the prevalence of upper extremity and neck among college students was reasonably high (Kalirathinam et al., 2017; Vate-U-Lan, 2015). 
It is undeniable the smartphone has become a necessity in everyday life for most people. Innovation in smartphones has contributed to rapid social changes through fulfilling personal life practices. Excessive use of smartphones, however, can lead to compulsive, repeated and constant head and neck movement toward the device all day long (Maharani \& Dewi, 2019). These situations resulted in damaging symptoms such as neck pain, shoulder pain, chronic headaches and increased spine curvature (Samani et al., 2018; Vate-U-Lan, 2015).

The word "text neck" used to describe neck pain that occurs in people who spend long periods using unstable electronic devices like smartphones and tablets with an incorrect body posture (Sangyong Lee, Lee, \& Park, 2015; Portelli \& Reid, 2018). To avoid such health risks, users need to keep their smartphones eyelevel, change their posture regularly and reduce smartphone use times. Although many users may not understand these risks and even if some users realize the dangers, others may not pay adequate attention to maintain a correct position when burying their smartphones (Shim, Lee, Lee \& Choi, 2013).

The goal of this research is to study the level of knowledge and understanding of text neck and neck pain among graduate students at USM and to develop an interactive mobile application to decrease the prevalence of text neck syndrome.

\section{LITERATURE REVIEWS}

\section{Text Neck Syndrome}

Dr Dean L. Fishman, a US chiropractor, coined the term "Text Neck." 'Text Neck' or another phrase turtle neck posture may be described as repetitive stress injury and long-term pain caused by excessive watching or texting on mobile handheld devices (Portelli \& Reid, 2018; Samani et al., 2018). The text neck directly affects the spine while flexing the head in different degrees forward. An upright posture, the ears align with the center of the shoulders, the weight of the average head experts is around 10 to $12 \mathrm{lbs}$ of force through the muscle of the neck. The importance of the head, however, will increase dramatically almost six times as much power can be generated, the same pressure as an average 8 year old baby or six 10-pin bowling ball when our head is moved one inch away from the neutral position (Portelli \& Reid, 2018; Sunil Neupane, U T Ifthikar Ali, 2017; Vate-U-Lan, 2015). 
The main factor leading to Text Neck prevalence is the excessive use of handheld mobile electronic devices, particularly smartphones. Computer technology has profoundly influenced the contemporary lifestyle of the people (Sojeong Lee, Kang \& Shin, 2015; Maharani \& Dewi, 2019).

However, excessive use of smartphones can lead to compulsive, repeated and constant head and neck movement toward the device all day long (Maharani \& Dewi, 2019). Users could hold the phone with both hands and type texts using both thumbs when typing text messages using the touch smartphone. They may have lowered the smartphone to reduce biomechanical loads on the shoulder joint and minimize fatigue development in the neighbouring muscles in two-handed use, resulting in a massive head flexion angle to look down on the smartphone (Sojeong Lee et al., 2015).

Studies have shown that nearly 75 per cent of the world's population spends an average of two to four hours a day reading small unsupported electronic computer screens (Kalirathinam et al., 2017; Portelli \& Reid, 2018). Users spend more than 20 hours a week emailing, playing sports, watching videos, texting, and using social network service (Sojeong Lee et al., 2015). A recent Hong Kong study suggests that the prevalence of musculoskeletal problems with smartphone use ranges from 17.3 per cent to 67.8 per cent for neck complaints among 1,049 people surveyed, 70 per cent are adults, and 30 per cent are children and adolescents (Kalirathinam et al., 2017; Samani et al., 2018).

Statistics also indicate that mobile use is more prevalent among both college and university students. Approximately 71.2 per cent of university students in Saudi Arabia reported cervical pain, while in Malaysia, the prevalence of upper extremity and musculoskeletal neck symptoms was relatively high among college students resulting in the incidence of these symptoms as "typical" or common (Alzarea \& Patil, 2015; Kalirathinam et al., 2017). In summary, spending more time on disabled mobile devices resulted in the incidence of text neck and cervical spine pain being higher (Sangyong Lee et al., 2015). Text neck may cause many adverse symptoms, including neck pain, pain in the shoulder, upper back pain, persistent headaches, and increased spine curvature (Vate-U-Lan, 2015).

Several symptoms can be experienced by the patient or person to diagnose the text neck, which is a sharp pain in the lower neck stages, feeling soreness at one spot or area of the neck and trapezoidal muscle region, feeling pain radiating from the neck into the shoulder and arms, numbness and weakness of the shoulder muscles and stiffness of the headache (Sunil Neupane, U T Ifthikar Ali, 2017; 
Vate-U-Lan, 2015). If a text neck is not treated or corrected promptly, it can result in severe permanent damage such as inflammation of the neck ligaments, muscles and nerves leading to permanent arthritic changes, spinal misalignment, spinal degeneration, disc compression and herniation, and spinal curve flattening (Samani et al., 2018; Sunil Neupane, U T Ifthikar Ali, 2017). The more time spent on forwarding head positioning, the more likely it is to have issues with the neckshoulder. With frequent break-away, the text neck could be avoided while using mobile devices, plus users need to keep the device at eye level too (Samani et al., 2018; Shim et al., 2013). While many users may not understand these risks, and even if some users realize the risks, most may not pay adequate attention or lack of knowledge to maintain a correct position when burying their smartphones (Samani et al., 2018; Shim et al., 2013; Vate-U-Lan, 2015).

Frequent breaks help to alleviate neck and shoulders tension (Khan et al., 2018; Sunil Neupane, U T Ifthikar Ali, 2017).

Users should follow the 20-20-20 rule, meaning they take a 20 -second break every 20 minutes and center the eyes on something at least 20 feet away to prevent a text neck (Vate-U-Lan, 2015). Clinically, several previous studies have provided evidence-based methods for text neck intervention (Kalirathinam et al., 2017; Vate-U-Lan, 2015), among which the McKenzie method is a representative therapeutic and diagnostic tool used by physical therapists to treat spinal pain ( $\mathrm{J}$. H. Lee et al., 2017). The details of the six moves are below:

1. Chin Tuck lying. Use your fingers to hold your chin and gently press back for 5 seconds before returning to the initial position.

2. Extension of the sitting neck. Slowly pull the chin backward and extend the head for 5 seconds before going back to the initial position.

3. Side-bend. Place one hand on the back neck and comfortably fasten the neck or knee while using another side to pull the head in the opposite direction.

4. Neck spinning. Pull the chin backward and rotate the head for 5 seconds before going back to the starting position.

5. Sitting flexion neck. Place one hand at the back of the neck and fix the neck while using another side to move the head forward for 5 seconds gently.

6. Neck stretch and rotation. Pull the chin backward and alternately extend and rotate the head for 5 seconds before returning to the initial position. 


\section{Related Work}

There were several attempts to solve the problem above. Using external devices or equipment attached to the body, the most solution has been required (Gupta, 2018; Ning et al., 2015; Shim et al., 2013). Using NexerciseVR, a Virtual Reality (VR) program, to promote user encouragement by delivering and engaging game-like experience during immersive exercise (Kim et al., 2019); Hermanis et al. (2013) received Biofeedback with a Wearable Posture Monitoring System over a smartphone.

Any time an unwanted position is detected, the user will be warned to perform restorative posture themselves. All of the features were in real-time, but the ease of use and comfort is reduced (Hermanis et al., 2013; Kim et al., 2019). Lee et al. have developed app-based neck exercises in another implementation that apply the McKenzie neck exercise to treat pain to the user (J. H. Lee et al., 2017). The app base neck exercise is equipped with a self-feedback function to increase compliance, satisfaction, and motivation of the users.

Also, some researchers used the built-in accelerometer, orientation sensor, and front camera of the smartphone to monitor the user's body position (Shim et al., 2013). However, the use of two internal smartphone sensors - the triaxial Accelerometer and Magnetic Sensor (compass) to get the phone's orientation (in degrees) through an Android library function — is a drawback: TYPE ORIENTATION. This function is, unfortunately, now deprecated and cannot be used (Gupta, 2018; Shim et al., 2013). If the. getOrientation new feature is called in its place, if the magnetic sensor is not found, no value will be returned. This is a significant downside that restricts the use of the application because it does not work in smart.

Moreover, the research suggested that to test if the user uses the phone, their device, Smart Pose, regularly checks the user's face from the front camera's captured images. However, capturing images using the front camera is not possible or ethically within Android Development limits without running them in real-time (Gupta, 2018). The limitation generally lies in the external equipment and the user's ease of use and comfort while using the application. Moreover, a proper warning system is absent from the solution. Due to the disparity between the qualities of smartphone cameras today, the app may not work reliably either.

This research paper presets Necks Up, overcoming most of the constraints. Increased reality will be used to reduce user costs for external devices as well as 
inconvenience and uncomfortability (Ning et al., 2015). Necks Up will gamify the neck exercise with AR to release the tension of the user's neck and help the user remain engaging and motivated. A notification shall be issued to the user whenever the user inappropriately uses the smartphone. The information will automatically delete hones that lack a magnetic sensor when the posture is corrected.

\section{Gamification}

Gamification can be defined as incorporating the element of gameplay into a non-game context (Thiel, 2016; Wong \& Kwok, 2016). It is also called turning an existing structure into a game, encouraging players to take part (Pernencar et al., 2018; Wong \& Kwok, 2016).

Nintendo's Pocket Pickachu and Ring-Fit adventure are the best examples of gamification while motivating players to engage by virtual character and video games in physical activity (Gil, 2020). The idea of incorporating gamified elements in a health-related application is to motivate users to continue to do exercise. Competition, progress bar, award, score, leader board, rule and feedback, and the theme of story are among the most common gamified elements. Wong \& Kwok (2016) summarized a gamified design feature research model that serves as a motivating affordance in physical activity, becoming a critical factor in healthrelated applications. NecksUp will be taking a challenge and social recognition as the essential element for game design for this research.

Challenge in gamified features served as gamification based on rewards includes accomplishment badges, points, and levels (Wong \& Kwok, 2016). The point system is primarily an essential element of all gamified systems. Liu et al. suggested that the affordance of competence could be treated as "individual games" (Thiel, 2016; Wong \& Kwok, 2016). For this research, the interface design allows users to set their targets rather than the general "one-size-fits-all" target. Therefore, users are motivated to break away from their regular heavy use of smartphone habits and exercise their neck towards their personal goals. When the users hit a specific target, they will instantly receive a notification with a particular accomplishment and constructive feedback to improve the incentive of the user to achieve their goals — social acknowledgment.

A similar button is designed to help users show their love and cares for others, and the "like" number is viewed at the support level of others. A similar function is defined by Hamari and Koivisto as an indicator of social influence and positive recognition (Wong \& Kwok, 2016). A study also indicates that a 
mobile health app requires social control and should be designed to support users (Wong \& Kwok, 2016). The games with the most success rely heavily on social interaction (Thiel, 2016).

\section{Augmented Reality}

Augmented Reality (AR) is a technology that makes it possible for users to experience the real-life world right before us. It differs from virtual reality (VR), as AR uses a camera on a device to add digital elements to a live view. At the same time, VR implies an experience of complete immersion that shuts out the physical world (Peddie, 2017). AR systems come with several wearables and non-wearable taxonomies (Peddie, 2017). Wearable devices include helmets, headsets, contact lenses, or smart glasses, and these wearable devices require a system and design that is more complex than non-wearable devices.

Meanwhile, accessing AR with non-wearable devices like smartphones, tablets, projectors, television, is much more comfortable. NecksUp is an AR-based mobile application in this research that combines face detection technology to create a gamification neck exercise to help users break away from their heavy smartphone use. Facial detection will be further discussed.

Smartphones have become extremely popular in recent years; it's changed AR's appearance. Equipped with a combination of computing power, camera, video display, and additional features such as GPS, network, touch, and civilian pricing, research on using smartphones as an AR platform has increasingly evolved (Peng, 2015).

Qualcomm Connected Experience Inc. is a product of QCAR (Vuforia). It's the software platform that allows for augmented reality app experiences across the most real-world environments, giving the power to see mobile apps. With IOS, Android, and Unity 3D support, QCAR allows a developer to write a single native app that can reach the most users across the smartphone and tablet widget range.

QCAR's smart Terrain technology for smartphones and tablet PCS is a breakthrough in the field of experiencing augmented reality. It will make the virtual characters have an unparalleled opportunity to communicate with the physical world, and it gives users a brand new level of interaction and practical experience (Peng, 2015). Face filter and snappable from Snapchat are the best examples for consumers to use face detection and AR to create a mask on their 
faces (Constine, 2018; Tommy, 2020). For instance, Snappables is an AR game that uses touch, motion, and facial expressions to compete for high scores or to head multiplayer matchups in a literal head (Constine, 2018).

\section{Face Detection}

Face detection finds faces information in the video sequence or image and determines the size, position, trajectory, attitude and further process of extraction of the human face eyes, lips and other features (Kitanovski \& Izquierdo, 2011; Peng, 2015); Introduction of face recognition technology began in the early 80's and 90's, focused primarily in still photographs. Technology advancement has brought more research focused on detecting the video stream in the real-time face. The intent to try determines quickly and accurately whether there was a face under the complex background in the video image sequence. When a face is present it gives the facial details, it monitors human faces dynamically. This is a type of operation for pattern recognition according to the characteristic features of face sample mode (Peng, 2015). In Peng's research, it is possible to realize the combination of face detection technology and mobile augmented reality in mobile AR applications. However, the drawback lies in the low image quality and graphical processing power (Peng, 2015). With the continuous development and enhancement of mobile terminal technology, smartphones have now become part of everyday life for people and the combination of face detection technology and AR technology has become common in our lives (Tommy, 2020).

\section{PROBLEM STATEMENTS}

Text neck is a chronic injury, which should be prevented by using smartphones with regular break-away. However, most smartphone users didn't ignore or disregard break-away to relax while using their device. This is possibly due to a lack of information and understanding of the term neck (Khan, Gillani, Khan, \& Wahid, 2018; Samani et al., 2018).

There were several attempts to solve the problem above. Most solutions used devices externally attached to the body (Gupta, 2018; Kim, Jeon, \& Moon, 2019; Ning, Huang, Hu, \& Nimbarte, 2015) have been required. Some researchers also used the built-in accelerometer of the smartphone to collect data and monitor user activity to avoid incorrect posture and neck pain (Shim et al., 2013). 
Most solutions have used external wearable devices to track daily activity via smartphone with real-time Biofeedback (Hermanis, Nesenbergs, Cacurs, \& Greitans, 2013).

While all of the features may increase user compliance, satisfaction, and motivation, the ease of use and comfort has significantly decreased (Hermanis et al., 2013; Kim et al., 2019); The goal of this project is therefore to develop an interactive mobile application, equipped with Augmented Reality (AR) and Face Detection technology to improve the knowledge and awareness of the text neck among undergraduate students.

\section{RESEARCH METHODOLOGY}

The research will adopt the quantitative investigation method in this study. Sources of quantitative data include observation and observation of participation (fieldwork), questionnaires, experiments, and analysis of content.

The method of questionnaires was employed for this research. The survey is a means to gage USM undergraduate students ' awareness and knowledge of text neck syndrome and opinions on the development of mobile application design. Most previous research and writings on Text Neck Syndrome have adopted a quantitative approach. Hence the researcher intended to follow a similar technique of primary data collection in this research. As the study of recognition and understanding of text neck syndrome is closely related to the field of medical science, several researchers from the similar area strongly endorse the concept of quantitative process analysis (Samani et al., 2018; Vate-U-Lan, 2015).

For the selection of participants, the researcher used the simple random sampling method. Simple random sampling refers to the equal chance that each member of the population will be selected. This means that the researcher selects participants at random to participate in the study. Similarly, this strategy allows the researcher to gather relevant and useful information to answer the research question. Because of the COVID 19 pandemic, the online survey was conducted via email and social media.

The data collected from the respondents will be arranged and summarized according to the categories mentioned above: demography, knowledge and awareness of the syndrome of the text neck, and data concerning the design of the mobile application. Following the study objectives, the researcher then analyzed and interpreted the information provided by the informants. 


\section{RESULTS}

To determine the response obtained from the population, descriptive surveys were carried out. For each question the percentage of answers has been determined. These data are used as reference for the development of design.

\section{What kind of phone do you have?}

20 responses

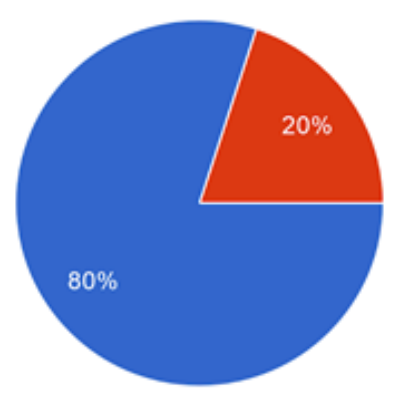

Android

Figure 1 Showing results on a question, what kind of phone do you have?

The diagram indicates what kind of telephone respondents possess. 80 per cent of those surveyed own Android smartphones, while just 20 per cent own iPhones.

This diagram below shows the daily average time spent by respondents on their smartphone.

How much time you spend on your smartphone?

20 responses

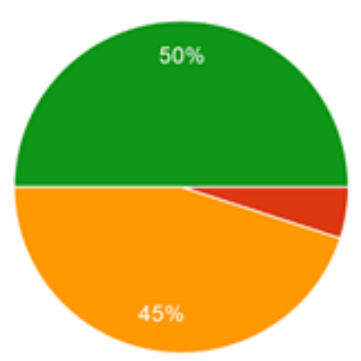

Less than one hour

1-2 hours

3. 5 hours

6 hours and above

Figure 2 Showing results on questions, how much time you spend on your smartphone? 
The chart shows that 50 per cent of respondents spend more than 6 hours on the smartphone, while only 5 per cent spend almost one to two hours on the smartphone and 45 per cent spend three to five hours on the smartphone. However, 0 per cent of respondents spend less than an hour on smartphones.

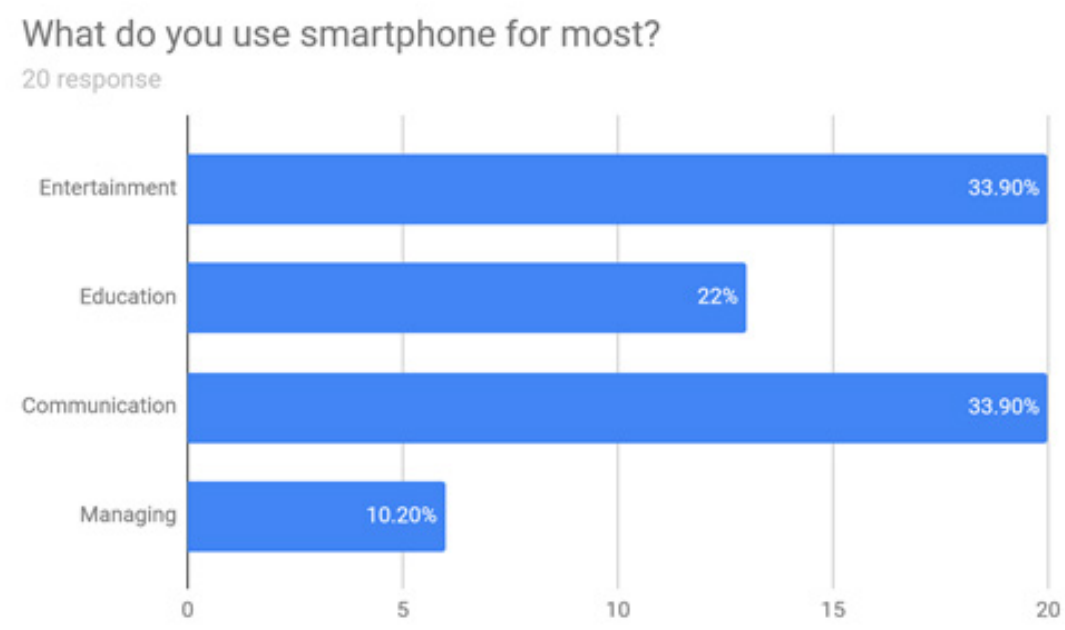

Figure 3 Showing results on questions. What do you use smartphones for most?

Figure 3 shows the categories most of which 20 respondents are using smartphones. The graph shows that 20 respondents spend 33.9 per cent on entertainment and communication smartphones, while only 22 per cent spend on education and 10.2 per cent on management.

Have you heard of text neck syndrome?

20 responses

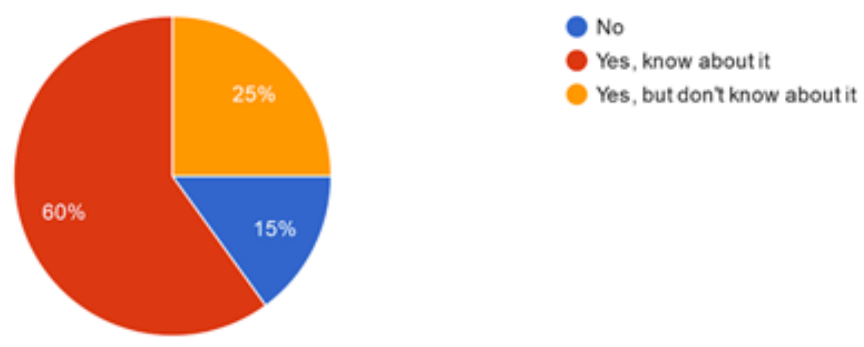

Figure 4 Showing results on questions, have you heard of text neck syndrome?

The chart shows 60 per cent of respondents heard of and are aware of text neck syndrome, 25 per cent of respondents heard of it but did not know about 
text neck syndrome, and only 15 per cent of respondents do not hear about text neck syndrome.

\section{Do you know how to prevent neck pain or text neck syndrome?}

20 responses

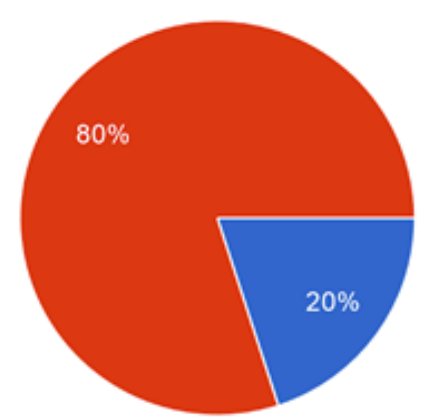

Figure 5 Showing results on question, do you know how to prevent neck pain or text neck syndrome?

Figure 5 indicates that only 20 per cent of respondents know how to avoid neck pain or syndrome of the text arm, while 80 per cent of respondents do not know the prevention.

\section{How often you occur neck pain while using smartphone in a week?}

20 responses
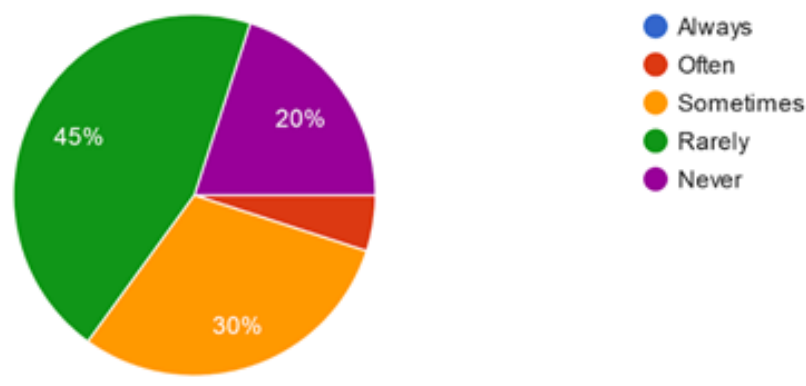

Figure 6 Showing results on questions, how often you occur neck pain while using smartphone in a week?

Figure 6 shows the frequency in which respondents experience neck pain while using a smartphone within a week. 45 per cent of respondents seldom experience neck pain when using a smartphone, when 30 per cent of respondents 
often experience it, and only 5 per cent of respondents often experience neck pain. However, 20 per cent of respondents never experience neck pain.

\section{Do you know any app that monitors/prevent neck pain or text neck syndrome? 20 responses}

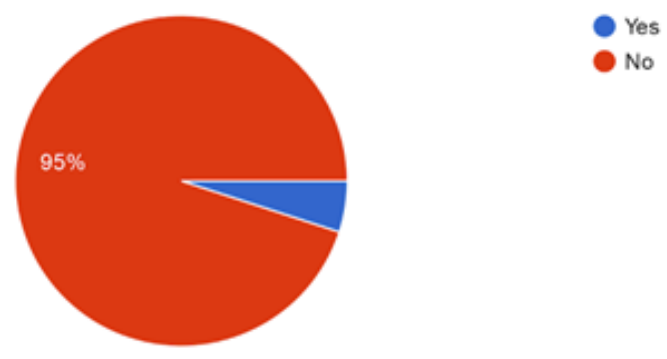

Figure 7 Showing results on question, do you know any app that monitors or prevents neck pain or text neck syndrome?

Figure 7 reveals that only 5 per cent of respondents are aware of any mobile application that tracks or prevents on the market neck pain or text neck syndrome while 95 per cent of respondents are unaware of this.

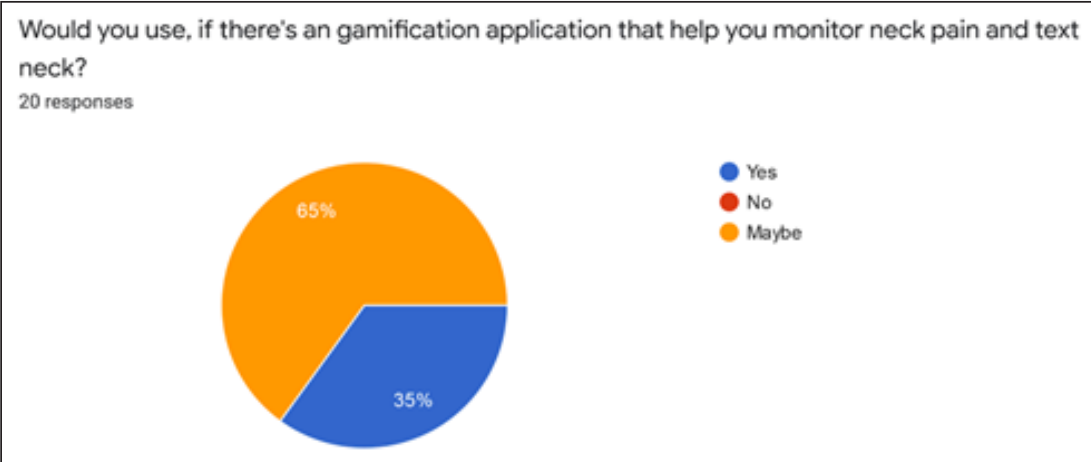

Figure 8 Showing results on questions, would you use, if there's an gamification application that helps you monitor neck pain and text neck syndrome?

Figure 8 shows that 65 per cent of respondents may try the gamification application that monitors neck pain and text neck syndrome, and 35 per cent will use the application with confidence. Yet the program will not be used by 0 per cent of respondents. 


\section{CONCLUSIONS}

Based on the findings in the level of knowledge and awareness of the text neck among undergraduate students of Universiti Sains Malaysia (USM), it can be concluded that the use of AR-based neck exercise gamification that alerts incorrect posture using a smartphone front camera and a built-in sensor could be developed to enhance levels of knowledge amongst hand phone users. The advanced technology enables people who are suffering from, or at risk of, text neck to have more pleasant and engaging neck exercise experience with a better exercise impact, and may promote regular break-away from the wrong posture.

\section{REFERENCES}

Alzarea, B. K., \& Patil, S. R. (2015). Mobile Phone Head and Neck Pain Syndrome: Proposal of a New Entity. Ohdm, 14 (5), 313-317.

Constine, J. (2018). Snapchat launches AR selfie games called Snappables $\mid$ TechCrunch. Retrieved June 3, 2020, from Techcrunch website: https://techcrunch. com/2018/04/25/snappables-snapchat-games/

Gupta, H. (2018). Smartphone based cervical spine stress prevention. Journal of Software Engineering and Applications, 11 (02), 110-120. https://doi.org/10.4236/ jsea.2018.112006

Hermanis, A., Nesenbergs, K., Cacurs, R., \& Greitans, M. (2013). Wearable posture monitoring system with biofeedback via smartphone. Journal of Medical and Bioengineering, 2 (1), 40-44. https://doi.org/10.12720/jomb.2.1.40-44

Kalirathinam, D., Manoharlal, M. A., Mei, C., Ling, C. K., Sheng, T. W. Y., Jerome, A., \& Mahadeva Rao, U. S. (2017). Association between the usage of smartphone as the risk factor for the prevalence of upper extremity and neck symptoms among university students: A cross-sectional survey based study. Research Journal of Pharmacy and Technology, 10 (4), 1184-1190. https://doi.org/10.5958/0974360X.2017.00213.X

Khan, A. F., Gillani, S. F. U. H. S., Khan, A. F., \& Wahid, A. (2018). Are you suffering pain neck due to smart phone text neck syndrome. Pakistan Journal of Medical and Health Sciences, 12(3), 1095-1097.

Kim, W., Jeon, I., \& Moon, J. (2019). NexerciseVR: A VR-based Exergame for Neck Exercise. SSRN Electronic Journal, 1. https://doi.org/10.2139/ssrn.3448344

Kitanovski, V., \& Izquierdo, E. (2011). 3D Tracking of Facial Features for Augmented Reality Applications. UK: Multimedia and Vision Research Group, Queen Mary, University of London.

Lee, J. H., Lee, M. Y., Lim, T. H., Kim, T. Y., Kim, S. M., Suh, D. W., ... Yoon, B. C. (2017). Effectiveness of an application-based neck exercise as a pain management tool for office workers with chronic neck pain and functional disability: A pilot randomized trial. European Journal of Integrative Medicine, 12 (April), 87-92. https://doi.org/10.1016/j.eujim.2017.04.012 
Lee, Sangyong, Lee, D., \& Park, J. (2015). Effect of the cervical flexion angle during smart phone use on muscle fatigue of the cervical erector spinae and upper trapezius. Journal of Physical Therapy Science, 2 (6), 1847-1849. https://doi. org/10.1589/jpts.27.1847

Lee, Sojeong, Kang, H., \& Shin, G. (2015). Head flexion angle while using a smartphone.

Ergonomics, 58 (2), 220-226. https://doi.org/10.1080/00140139.2014.967311

Maharani, D. P., \& Dewi. (2019). The Effect of Duration of Smartphone Usage and Level of Smartphone Addiction, 2 (11), 32-35.

Ning, X., Huang, Y., Hu, B., \& Nimbarte, A. D. (2015). Neck kinematics and muscle activity during mobile device operations. International Journal of Industrial Ergonomics, 48, 10-15. https://doi.org/10.1016/j.ergon.2015.03.003

Palladino, T. (2020). Snapchat Turns Real World into AR Scavenger Hunt with Nearly Instant Object Recognition, Mobile AR News: Next Reality. Retrieved June 3, 2020, from https://mobile-ar.reality.news/news/snapchat-turns-real-world-intoar-scavenger-hunt-with-nearly-instant-object-recognition-0230749/

Peddie, J. (2017). Augmented Reality: Where we all live. Springer International. https:// doi.org/10.1007/978-3-319-54502-8

Peng, H. (2015). Application research on face detection technology based on OpenCV in Mobile Augmented Reality. International Journal of Signal Processing, Image Processing and Pattern Recognition, 8(4), 249-256. https://doi.org/10.14257/ ijsip.2015.8.4.22

Pernencar, C., Sousa, P., Frontini, R., Martinho, R., Runte, D., Mendes, D., \& Carvalho, M. (2018). Planning a health promotion program: Mobile app gamification as a tool to engage adolescents. Procedia Computer Science, 138, 113-118. https:// doi.org/10.1016/j.procs.2018.10.016

Portelli, A., \& Reid, S. A. (2018). Cervical proprioception in a young population who spend long periods on mobile devices: A 2-Group comparative observational study. Journal of Manipulative and Physiological Therapeutics, 41(2), 123-128. https://doi.org/10.1016/j.jmpt.2017.10.004

Samani, P. P., Athavale, N. A., Shyam, A., \& Sancheti, P. K. (2018). Awareness of text neck syndrome in young-adult population. International Journal of Community Medicine and Public Health, 5(8), 3335. https://doi.org/10.18203/2394-6040. ijcmph20183057

Shim, E., Lee, S., Lee, H., \& Choi, Y. S. (2013). Smart Pose: Mobile Posture-aware System for Lowering Physical Health Risk of Smartphone Users. Conference on Human Factors in Computing Systems - Proceedings, 2013-April, 2257-2266. https://doi.org/10.1145/2468356.2468747

Sunil Neupane, U T Ifthikar \& Ali, M. A. (2017). Text Neck Syndrome - systematic review. Imperial Journal of Interdisciplinary Research, 3 (7), 141-148.

Thiel, S. K. (2016). Reward-based vs. Social gamification: Exploring effectiveness of gamefulness in public participation. ACM International Conference Proceeding Series, 23-27, Octo. https://doi.org/10.1145/2971485.2996739

Vate-U-Lan, P. (2015). Text Neck epidemic: A growing problem for smart phone users in Thailand. International Journal of the Computer, the Internet and Management, 23 (3), 27-32. 
What is a Mobile Application? - Definition from Techopedia. (n.d.). Retrieved June 3, 2020, from https://www.techopedia.com/definition/2953/mobile-applicationmobile-app

Wong, C. C. K., \& Kwok, R. C. W. (2016). The effect of gamified mHealth app on exercise motivation and physical activity. Pacific Asia Conference on Information Systems, PACIS 2016. 